\title{
Fountain's Sign as a Diagnostic Key in Acute Idiopathic Scrotal Edema: Case Report and Review of the Literature
}

\author{
Dimitrios Patoulias ${ }^{1, *}$, Vasileios Rafailidis ${ }^{2}$, Thomas Feidantsis ${ }^{3}$, Maria Kalogirou4, Dimitrios Rafailidis ${ }^{5}$, \\ Ioannis Patoulias ${ }^{3}$
}

\begin{abstract}
The acute idiopathic scrotal edema (AISE) is a self-limited disease of unknown etiology, characterized by edema and erythema of the scrotum and the dartos, without expansion to the underlying layers of scrotum's wall or to the endoscrotal structures. Boys younger than 10 years old are usually involved in $60-90 \%$ of all cases. Diagnosis is made after exclusion of other causes of acute scrotum. We present a case of a 7-year old boy, who was admitted to the Emergency Department due to development of scrotal edema and erythema over the last 48 hours, which extended to the base of the penis. The patient mentioned that he first noticed the erythema on the anterior surface of the right hemiscrotum, which gradually extended. Physical examination did not reveal presence of pathology involving the endoscrotal structures, indicative of need for urgent surgical intervention. Transillumination was negative for blue dot sign. Ultrasonographic examination of the scrotum documented the homogeneity of the testicular parenchyma, while color Doppler revealed the presence of fountain's sign (equal arterial blood supply to both testicles). Conservative strategy was followed and the patient gradually improved within the next three days.

In conclusion, meticulous physical examination along with ultrasonographic examination of the suffering scrotum, especially with the highlighting of fountain's sign with color Doppler, document the diagnosis of AISE. Thus, need for urgent surgical investigation of the suffering scrotum due to diagnostic doubt is limited.

\section{KEYWORDS}

acute idiopathic scrotal edema; Fountain's sign; color Doppler; endoscrotal structures

AUTHOR AFFILIATIONS

${ }^{1}$ Department of Internal Medicine, General Hospital of Veria, Veria, Greece

2 Department of Radiology, Aristotle University of Thessaloniki, General Hospital 'AHEPA', Thessaloniki, Greece

${ }^{3}$ 1st Department of Pediatric Surgery, Aristotle University of Thessaloniki, General Hospital 'G. Gennimatas', Thessaloniki, Greece

${ }^{4}$ Health Center of Kalambaka, General Hospital of Trikala, Trikala, Greece

${ }^{5}$ Department of Radiology, General Hospital 'G. Gennimatas', Thessaloniki, Greece

* Corresponding author: M. Alexandrou 3B, Pefka, Thessaloniki, Postal code 57010; e-mail: dipatoulias@gmail.com
\end{abstract}

Received: 5 November 2017

Accepted: 27 March 2018

Published online: 2 July 2018

Acta Medica (Hradec Králové) 2018; 61(1): 37-39

https://doi.org/10.14712/18059694.2018.22

(c) 2018 The Authors. This is an open-access article distributed under the terms of the Creative Commons Attribution License (http://creativecommons.org/licenses/by/4.0), which permits unrestricted use, distribution, and reproduction in any medium, provided the original author and source are credited. 


\section{INTRODUCTION}

Of all the clinical entities that come under the term "acute painful scrotum" in childhood, $2.3 \%$ of those cases are attributed to AISE $(1,2)$. The age distribution of clinical manifestation of acute scrotal edema ranges from 5 to 11 years. In $60-90 \%$ of all cases, AISE manifests in boys 10 years old or less (1).

It is a self-limited disease of unknown etiology, characterized by edema and erythema of the scrotum and the dartos, without expansion to the underlying layers of scrotum's wall or to the endoscrotal structures. Typically, it starts from the groin or the perineum with rapid expansion to the scrotum (2).

Aim of the present case study is to make a suggestion regarding the need for substantial modification of diagnostic approach in potential cases of AISE. In other words, diagnostic documentation should not be made on the basis of exclusion, but with the highlighting the crucial role of fountain's sign as a diagnostic key.

\section{CASE DESCRIPTION}

A 7-year old boy was admitted to the Emergency Department, due to development of scrotal edema and erythema over the last 48 hours, which extended to the base of the penis. The patient noticed that the erythema started from the anterior surface of the right hemiscrotum and gradually extended to the whole anterior surface of the scrotum and to the penile basis (Figure 1).

Meticulous physical examination did not reveal findings indicative of pathology of endoscrotal structures. Both testicles were of equal size, painless and orthotopic, with vertical orientation and normal turgidity. The patient complained for pain during the palpation of the right testicle, however, this was not a stable finding. Cremasteric muscle reflex was produced bilaterally. Palpation of epididymis was painless bilaterally, without observation

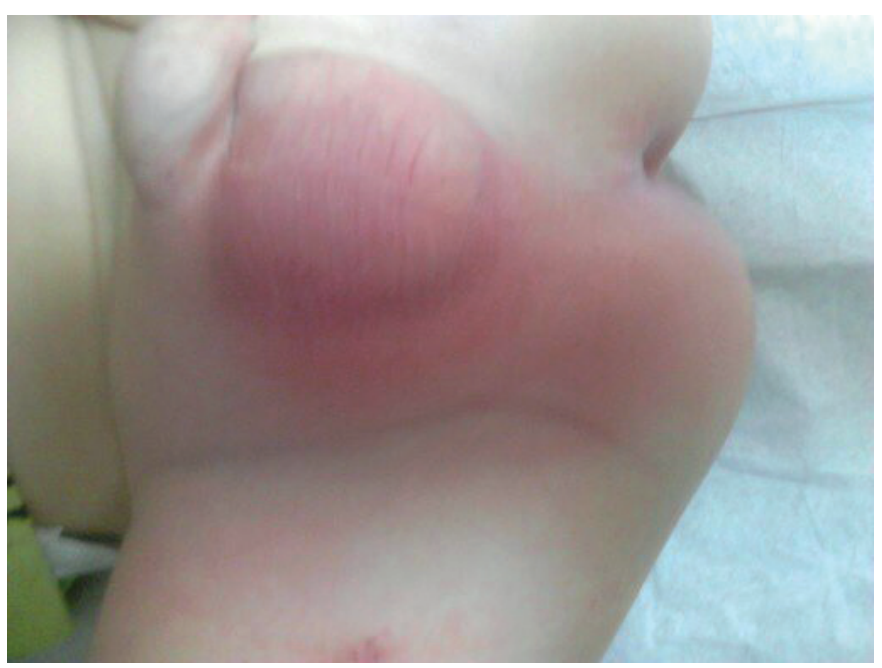

Fig. 1 Notice the erythema and the edema on the anterior surface of the right hemiscrotum, which gradually involved the anterior surface of the entire scrotum.

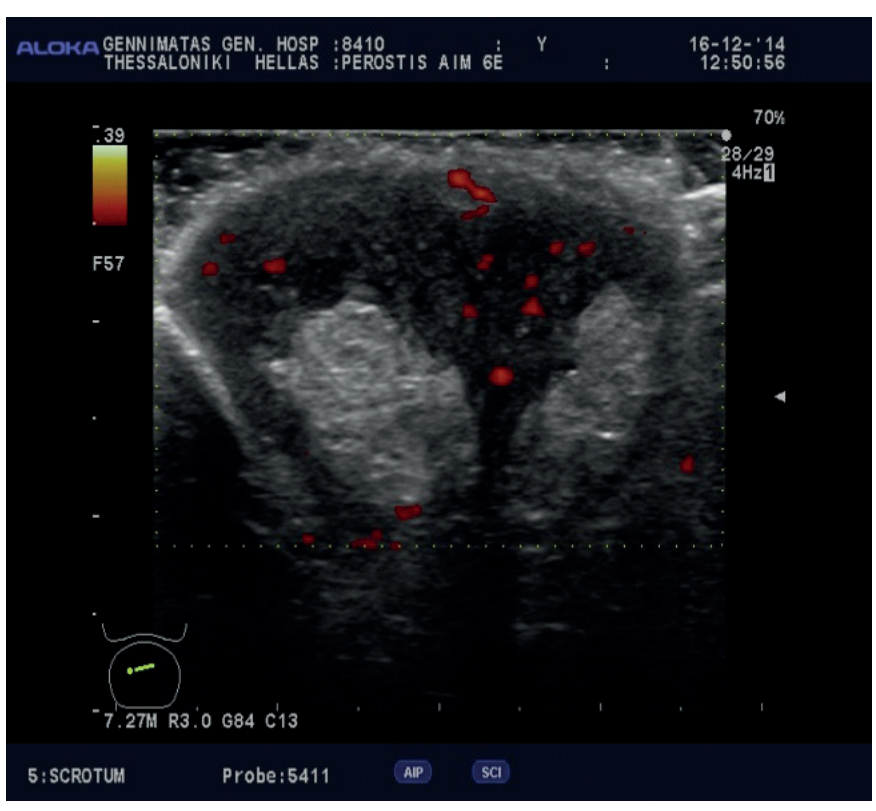

Fig. 2 Fountain's sign, as depicted in color Doppler examination in our case.

of edema, while Prehn's sign was negative. Blue dot sign also turned out negative during transillumination.

Ultrasonographic examination of the suffering scrotum followed and revealed the homogeneity of the testicular parenchyma, while we also measured the thickness of the scrotal wall, which was $7.2 \mathrm{~mm}$. Color Doppler documented the equal arterial blood supply to both testicles, finding consistent with fountain's sign (Figure 2).

As there was no clinical or imaging indication of surgical investigation of the suffering scrotum, we decided to treat our patient conservatively. He actually improved within the next three days after admission. He was discharged home after the 3rd day of hospitalization in good general condition. The patient was re-examined as an outpatient 10 and 20 days after admission, without evidence, both clinical and ultrasonographic, of pathology involving the endoscrotal structures.

\section{DISCUSSION}

The etiology of AISE is currently unknown, although certain situations have occasionally been implicated in the pathogenesis of this entity, such as:

1. The cellulitis, that extends from the perianal region to the scrotum. This approach lacks, because, on the one hand, the cultures received from the region are usually negative, and on the other hand, the AISE is mainly self-limited without therapeutic intervention.

2. The bites caused by insects.

3. An injury.

4. The urine loss, which causes aseptic inflammatory response in the area, expanding to the scrotum.

5. The allergy. It is considered as the most likely cause; based on the fact that eosinophilia is found in more than $40 \%$ of all AISE cases, while these patients also suffer from diseases such as asthma, eczema or contact 
dermatitis. Besides, there is evidence that medication with antihistamines contributes to the recession of the symptoms. Thus, it is believed that AISE is a clinical manifestation of angioedema $(3,4)$.

6. The acute Epstein-Barr viral (EBV) infection (5).

These patients usually do not complain of accompanying symptoms. They can rarely mention indistinct discomfort in the suffering scrotum. Rarely, itchiness precedes edema. Generally, scrotal edema is the first clinical manifestation; it develops suddenly and is accompanied with diffuse erythema in the affected area. AISE spreads rapidly, within a few hours. Edema most often begins unilaterally $(L / R=1)$, but it progressively extends to the whole scrotum. Erythema expands to the groin (67\% of all cases), the perineum (42\%), the penis $(20 \%)$ or to the suprapubic region. Edema and erythema are the major clinical manifestations of AISE, while local pain coexists in $80 \%$ of all cases. The duration of the above mentioned clinical manifestations usually ranges from 6 to 72 hours (average 14 hours) $(1,2)$. Soon begins the recession of edema and erythema, which is usually complete within 48 hours, without residual signs. In $21 \%$ of all cases, AISE recurs from 1 to 3 times, without complication. Relapse occurs several months or even years after the prior event.

Regarding differential diagnosis based upon physical examination, pathognomonic clinical signs in cases of testicular torsion are the absence of cremasteric muscle reflex and the high position of the testicle. In cases of appendiceal torsion, localized tenderness and pain during palpation of the upper pole of the suffering testicle and positive blue dot sign in transillumination of the suffering hemiscrotum are pathognomonic $(1,2,6)$. Finally, in acute epididymitis, pain during palpation of the suffering epididymis, along with the presence of edema, are typical.

Major imaging modality is color Doppler, as it contributes substantially to the exclusion or confirmation of other main causes of acute scrotum - most significantly, of those requiring urgent surgical intervention, soon after diagnosis -, and to the diagnostic documentation of AISE, with the highlighting of pathognomonic findings, including fountain's sign (7-10).

Exclusion of the testicular torsion can be made accurately, after conduction of high resolution ultrasound imaging with the use of frequencies $10-12 \mathrm{MHz}$ : basic indication of testicular torsion is the observation of rotations of the spermatic cord (snail-shell shaped mass), and not the disruption of the testicular circulation (11). High resolution ultrasound features $96 \%$ sensitivity, while color Doppler ultrasound is inferior, with $76 \%$ sensitivity $(11,12)$.

After performing cross sections, the homogenous thickening of the scrotal wall is documented, ranging from 3.4 to $13.4 \mathrm{~mm}$ (average 7.7-11.2 $\mathrm{mm}$ ), as in our case. A second pathognomonic finding is the increased vascularization of the scrotal wall, which is not always helpful in pediatric population $(10,13)$.

Blood supply to the scrotum is ensured by the anterior and posterior scrotal artery, which originate from deep branches of the internal and external pudendal artery. Depiction of those vascular branches in cross sections, along with observation of the scrotal hyperemia, constitutes the characteristic fountain's sign $(7,8,14,16)$. Other potential ultrasonographic findings are the development of reactive hydrocele, which occurs in $20 \%$ of all AISE cases, and the presence of swollen and hyperemic inguinal lymph nodes (1). None of the aforementioned findings was observed in our case.

\section{CONCLUSION}

Formerly, diagnosis of AISE was made via the exclusion of other causes of acute painful scrotum. Meticulous physical examination by the pediatric surgeon with precise interpretation of the clinical findings, along with the depiction of pathognomonic signs in color Doppler, and especially of fountain's sign, are crucial for the correct diagnosis. Thus, the necessity of urgent surgical investigation of the suffering scrotum due to diagnostic doubt - especially for the documentation or exclusion of testicular torsion - is significantly restricted.

\section{CONFLICT OF INTEREST}

None of the contributing authors have any conflict of interest, including specific financial interests or relationships and affiliations relevant to the subject matter or materials discussed in the manuscript.

\section{REFERENCES}

1. Klin B, Lotan G, Efrati Y, Zlotkevich L, Strauss S. Acute idiopathic scrotal edema in children - revisited. J Pediatr Surg 2002; 37(8): 1200-2.

2. Holcomb GW, Murphy JP, Ostlie DJ. In: Ashcraft's Pediatric Surgery Sixth Ed., Chapter: Acute Scrotum. Saunders Elsevier 2014, p. 705.

3. Van Langen AM, Gal S, Hulsmann AR, De Nef JJ. Acute idiopathic scrotal oedema: four cases and a short review. Eur J Pediatr 2001; 160(7): 455-6.

4. Weinberger LN, Zirwas MJ, English JC III. A diagnostic algorithm for male genital oedema. J Eur Acad Dermatol Venereol 2007; 21(2): 156-62.

5. Lee SH. Acute idiopathic scrotal edema caused by Epstein-Barr Virus. Pediatr Infect Dis J 2016; 35(5): 593.

6. Molokwu CN, Somani BK, Goodman CM. Outcomes of scrotal exploration for acute scrotal pain suspicious of testicular torsion: a consecutive case series of 173 patients. BJU Int 2011; 107(6): 990-3.

7. Breen M, Murphy K, Chow J, Kiely E, O’Regan K. Acute idiopathic scrotal edema (case report). Case Rep Urol 2013; 2013: 829345.

8. Geiger J, Epelman M, Darge K. The fountain sign: a novel color Doppler sonographic finding for the diagnosis of acute idiopathic scrotal edema, J Ultrasound Med 2010; 29(8): 1233-7.

9. Copertino M, Benelli E, Gregori M , Barbi E, Ventura A. A shining scrotal fountain. J Pediatr 2015; 167(1): 205.e1.

10. Mesquita RD, Rosas JL. Adult acute scrotal edema-when radiologists can help to avoid unnecessary surgical treatment. J Radiol Case Rep 2017; 11 (8): 24-30.

11. Aso C, Enríquez G, Fité M, et al. Gray-scale and color Doppler sonography of scrotal disorders in children: an update. Radiographics 2005; 25(5): 1197-214.

12. Carkaci S, Ozkan E, Lane D, Yang WT. Scrotal sonography revisited. J Clin Ultrasound 2010; 38(1): 21-37.

13. Lee A, Park SJ, Lee HK, Hong HS, Lee BH, Kim DH. Acute idiopathic scrotal edema: Ultrasonographic findings at an emergency unit. Eur Radiol 2009; 19(8): 2075-80.

14. Yusuf GT, Sidhu PS. A review of ultrasound imaging in scrotal emergencies. J Ultrasound 2013; 16(4): 171-8.

15. Roic G. Sonography of the acute scrotum in children. Pediatrics Today $2016 ; 12(1): 65-74$.

16. Tietze A, Avula S. Acute Idiopathic Scrotal edema. Euro Rad 2008; Case 6477. 